\title{
Original Research \\ Comparison of diagnostic accuracy of immediate angiography derived residual quantitative flow ratio after bioresorbable scaffold and drug eluting stent implantation
}

\author{
Zhengwei Li ${ }^{1,2}$, Jiachen Zhan ${ }^{1,3}$, Jia Han ${ }^{1}$, Guosheng Fu ${ }^{1,2, *, \dagger}$, Chongying Jin ${ }^{1,2, *, \dagger}$ \\ ${ }^{1}$ Department of Cardiology, Sir Run Run Shaw Hospital, Zhejiang University School of Medicine, 310016 Hangzhou, Zhejiang, China \\ ${ }^{2}$ Key Laboratory of Cardiovascular Intervention and Regenerative Medicine of Zhejiang Province, 310016 Hangzhou, Zhejiang, China \\ ${ }^{3}$ Department of Cardiology, Zhuji People's Hospital, 311800 Shaoxing, Zhejiang, China \\ *Correspondence: fugs@zju.edu.cn (Guosheng Fu); 3313019@zju.edu.cn (Chongying Jin) \\ ${ }^{\dagger}$ These authors contributed equally. \\ Academic Editors: Brian Tomlinson and Takatoshi Kasai \\ Submitted: 2 November 2021 Revised: 9 December 2021 Accepted: 13 December 2021 Published: 12 February 2022
}

\begin{abstract}
Background: Quantitative flow ratio (QFR) is a novel angiography derived fractional flow reserve (FFR) technique. However, its diagnostic accuracy has only be validated in native coronary lesions but not in vessels after bioresorbable scaffold (BRS) implantation. This study aims to evaluate the diagnostic accuracy of residual QFR in coronary vessels immediately post-BRS implantation. Methods: This is a retrospective, two center, validation cohort study. 73 stable angina patients who received at least one de novo lesion of an everolimus eluting stent (EES)/BRS implantation with subsequent residual FFR assessment were screened. Patients with aorta-ostial stenoses, bridge vessels at the distal segment of targeted vessels, acute coronary syndrome, previous coronary artery bypass grafting, age $<18$ years, lack of $\geq 2$ final angiographic projections were excluded. Contrast QFR assessment was performed blinded to FFR assessment. Results: A good correlation $(\mathrm{r}=0.680, p<0.001)$ was found between residual QFR and FFR. In the EES implantation cohort, a good correlation $(\mathrm{r}=0.769, p<0.001)$ was found between residual QFR and FFR, and a moderate correlation $(\mathrm{r}=0.446, p=$ 0.038 ) in the BRS cohort. The area under the Receiver operator characteristic (ROC) curve for detecting FFR $\leq 0.86$ was 0.883 for all patients. Conclusion: Residual QFR assessment after BRS implantation is feasible, and has a moderate correlation and agreement with residual FFR. QFR may be a promising tool similar to FFR to evaluate post-BRS effect.
\end{abstract}

Keywords: Quantitative flow ratio; Bioresorbable scaffold; Fractional flow reserve; Coronary de novo lesion; Everolimus eluting stent

\section{Background}

Percutaneous coronary intervention (PCI) is an effective technique to relieve myocardial ischemia [1]. Fractional flow reserve (FFR), an invasive technique using adenosine infusions, has been the gold standard to evaluate the functional significance of coronary artery lesions [2]. FFR $\leq 0.80$ indicates functionally significant stenoses, and predicts superior outcomes following PCI [3]. As a result, recent guidelines recommend FFR as a class IA recommendation [4]. Quantitative flow ratio (QFR) is a novel method to evaluate the functional significance of coronary stenoses using 3-dimensional angiographic reconstruction and fluid dynamics algorithms [5]. The FAVOR Pilot study showed promising results when QFR was used to identify functionally significant stenoses [5]. The favorable results of QFR do not require pharmacologic hyperemia with adenosine and decreases procedure time, risks, and costs. QFR was found to be superior to angiographic assessment for coronary artery stenoses in the FAVOR II Europe-Japan Study [6]. The FAVOR II China trial showed that QFR demonstrated high feasibility and accuracy [7]. The FAVOR III China study showed excellent outcomes with a QFR-guided PCI compared with an angiography-guided PCI strategy [8].
These studies demonstrated the feasibility and accuracy of QFR in assessing the severity of coronary stenoses. Diagnostic accuracy of residual QFR correlated well with post-PCI FFR [9]. To further evaluate the effect of postPCI, QFR was developed for invasive FFR approximation. In clinical practice, the success of a PCI is based solely on the post PCI angiographic assessment. Post-PCI physiologic assessments showed that up to $30 \%$ of patients had an FFR $<0.8$, especially in complex lesions where additional PCI may not be of further benefit based on Optical Coherence Tomography (OCT)/intravenous ultrasound (IVUS). This may lead to unnecessary additional interventions, resulting in increased costs and morbidity. Since QFR is able to assess the hemodynamic significance of a stenosis with high diagnostic accuracy $(92.7 \%$ in FAVOR II China study), this technique offers a non-invasive method to assess post-PCI efficacy.

With the development of PCI, the concept of nonimplantation is deeply rooted in the philosophy of cardiologists. BRS is a novel poly-L-lactic acid (PLLA) — based scaffold [10], whose beam is completely absorbed within 3 5 years after implantation. The thickness of BRS is $170 \mu \mathrm{m}$. Compared with EES, the beam of BRS is thicker, which reduces the residual vessel diameter, which may affect the 


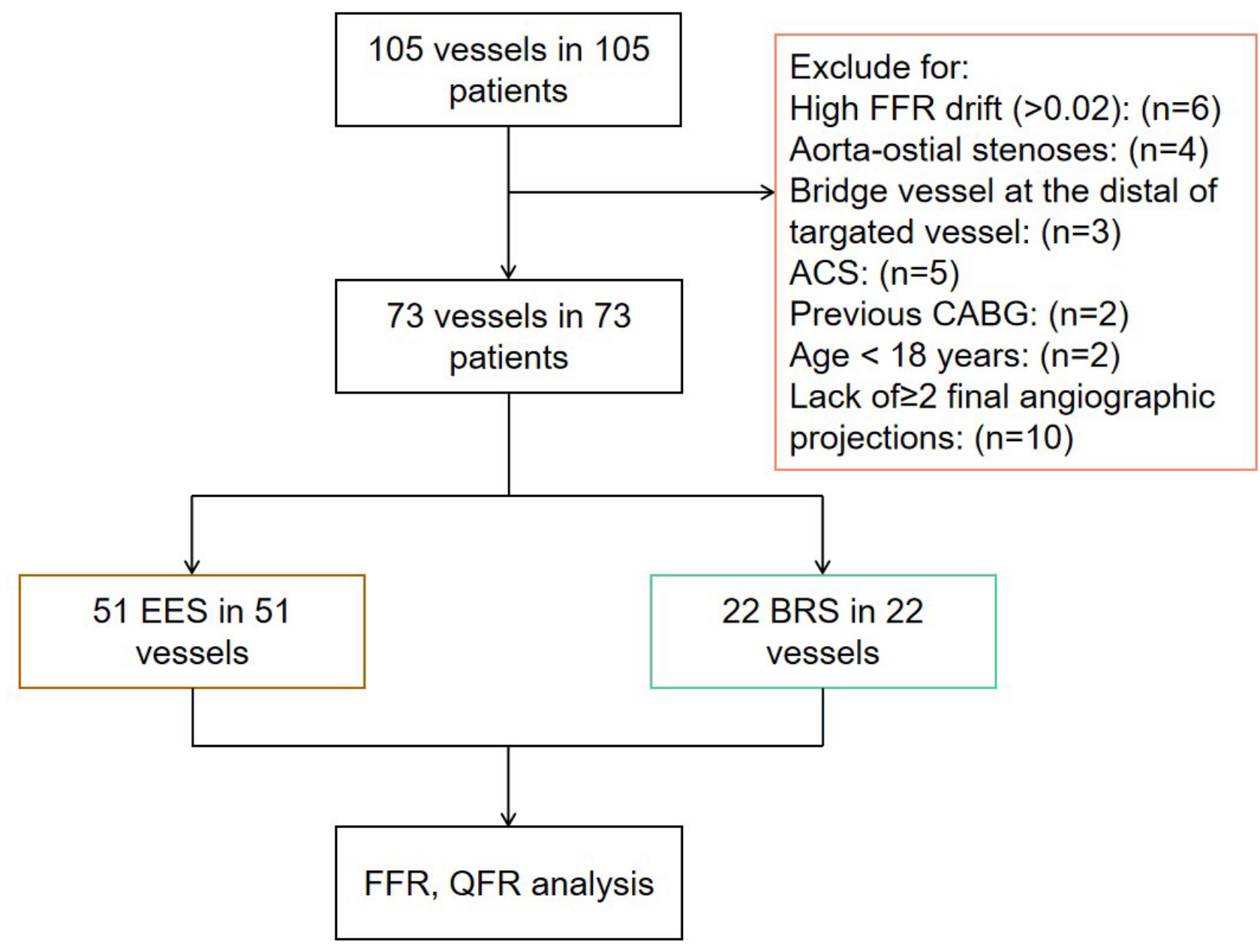

Fig. 1. Study flow chart. 105 patients underwent residual FFR measurements post-PCI. After screening for enrollment and exclusion criteria, 73 lesions from 73 patients were eventually enrolled into this study.

residual QFR post-BRS implantation. Currently, there have been no published studies on the evaluation of residual QFR post-BRS implantation. In this study, we evaluated the residual QFR post-BRS implantation compared with postEES implantation using residual FFR.

The aim of this study is to evaluate the diagnostic accuracy of residual QFR in coronary vessels immediately after BRS implantation. We assessed the relationship between residual QFR and FFR in a cohort of patients postEES or -BRS implantation.

\section{Methods}

\subsection{Study population}

Patients who were referred for PCI and then for invasive FFR measurement following stent implantation were included in this study. Patients were excluded if they had aorta-ostial stenoses, bridge vessels at the distal segment of the targeted vessel, acute coronary syndrome (ACS), previous coronary artery bypass grafting (CABG), age $<18$ years, and lack of $\geq 2$ final angiographic projections.

\subsection{Study design}

We conducted a retrospective, two center, validation cohort study from June 2019 to July 2020. During the study period, 105 patients underwent residual FFR measurements post-PCI as shown in Fig. 1. 6 patients were excluded for high FFR drift; 4 patients were excluded for aorta-ostial stenoses, 3 patients were excluded for bridge vessels at the distal targeted vessel, 5 patients were excluded for ACS, 2 patients were excluded for previous CABG, 2 patients were excluded for age $<18$ years, and 10 patients were excluded for lack of $\geq 2$ final angiographic projections. Contrast residual QFR assessment was performed blinded to the FFR assessment. 73 patients were included for analysis. 51 patients were implanted with EES, while 22 patients were implanted with BRS. A study flow chart is shown in Fig. 1. The matrix material of BRS was L-polylactic acid. The matrix material of EES was alloy. 

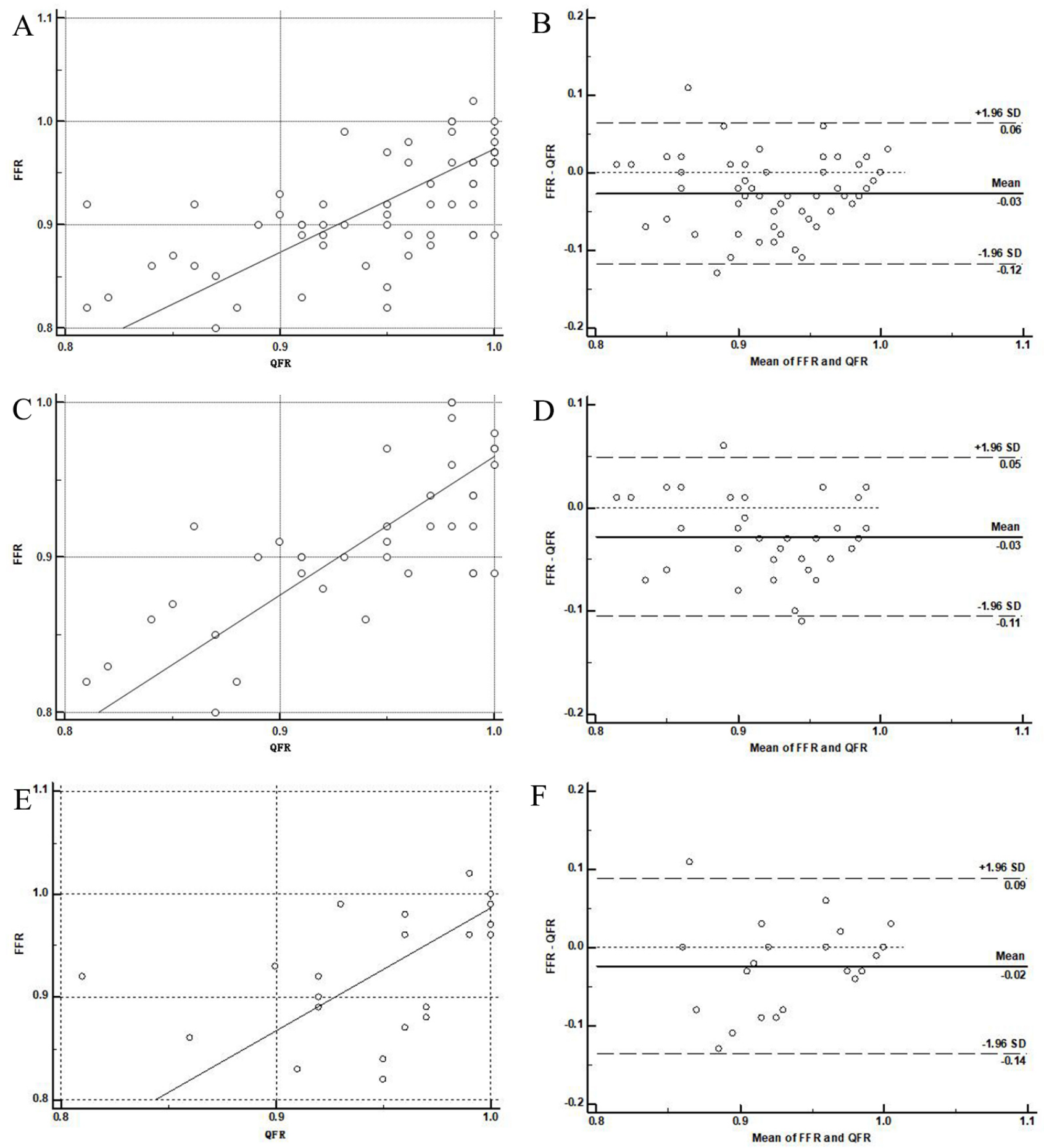

Fig. 2. Pearson's correlation. (A) and agreement (B) between QFR and FFR in 73 patients. Pearson's correlation (C) and agreement (D) between QFR and FFR in EES group. Pearson's correlation (E) and agreement (F) between QFR and FFR in BRS group.

\subsection{FFR measurement}

Angiographic projections were performed with biplane systems. All FFR measurements were performed with a pressure wire and a monitor (St. Jude Medical, Minn, USA). A bolus of $200 \mu \mathrm{g}$ nitroglycerin was administered intracoronary prior to measurements. FFR measurements were subjected to initial equalization with the pressure sen- sors at $1 \mathrm{~mm}$ out of the guiding catheter and recorded under fluoroscopy. Equalization and all FFR measurements were systematically performed after flushing with heparinized saline solution, and withdrawal of the wire needle of the Y-shaped connector. For invasive FFR measurements, the pressure wire was located far from the stent and maximal hyperemia was induced by continuous intravenous infusion 

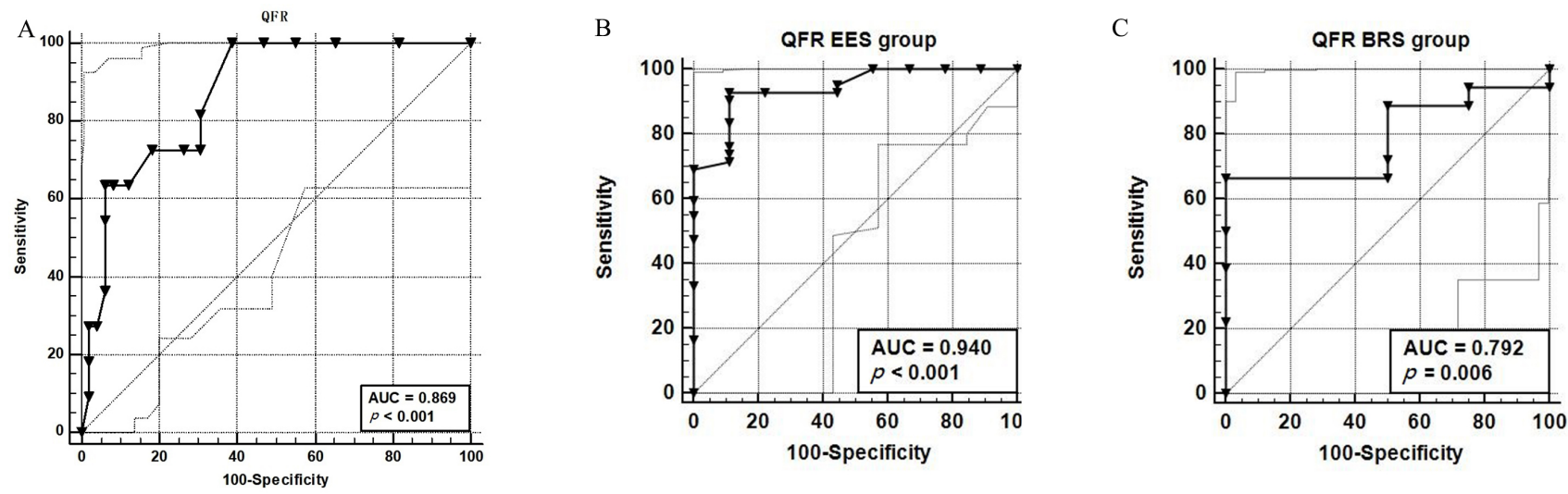

Fig. 3. Area Under Receiver-operating characteritic curve for QFR. ROC for QFR to detect an invasive FFR of $\leq 0.86$ in total patients (A). ROC for QRF to detect FFR $\leq 0.86$ in EES group (B) and BRS group (C).

of adenosine (140 $\mu \mathrm{g} / \mathrm{kg} / \mathrm{min})$. FFR was performed followed by stenting and then immediately afterward, a postPCI FFR was obtained.

\subsection{QFR calculation}

Details concerning the QFR calculation have been reported previously [11]. At least two adequate contrast-filled angiographic projections with $>25^{\circ}$ apart were acquired for QFR calculation. The observer calculated the contrast flow QFR values, unaware of the pressure-wire FFR value. The location of the FFR pressure-wire was identified at the angiographic projections and the QFR values were measured at the same location. For each vessel the flow models were applied to a 3D reconstruction of that vessel. QFR uses frame count analysis from regular (non-hyperemic) angiographic projections to model hyperemic flow velocity. For the invasive FFR and QFR models, all projections were acquired with 15 frames/s. None of the vessels with QFR $>0.86$ had a FFR value of $\leq 0.80$ for both QFR models.

\subsection{Statistical analysis}

Continuous variables were reported as mean \pm SD or median with interquartile range according to their distributions, which were checked by the Kolmogorov-Smirnov test. Categorical variables were reported as $\mathrm{n}(\%)$. The one-sample $T$ test was used to test whether the QFR and FFR values differed significantly from zero. Agreement between the QFR and FFR was assessed using Bland-Altman and correlation was determined by Pearson's correlation coefficient. Receiver operator characteristic (ROC) curves were compared using the DeLong method. All statistical analyses were performed with SPSS Statistics software (Version 20.0, NY, USA). A $p$ value $<0.05$ was considered statistically significant.

\section{Results}

\subsection{Patients characteristics}

In total, 73 vessels in 73 patients were included in the study. The baseline characteristics of all patients are shown in Table 1. 17 patients had diabetes mellitus and 40 patients had hypertension. 22 patients were smokers. 55 patients had a prior PCI. None of the patients had prior ACS, CABG or chronic obstructive pulmonary disease (COPD). 51 patients had been implanted with everolimus-eluting stents (EES) and 22 patients had been implanted with bioresorbable scaffold (BRS).

\subsection{Relationship between QFR and FFR}

In total, 73 coronary arteries were analyzed. The mean residual QFR and FFR values was $0.91 \pm 0.06$ and $0.94 \pm$ $0.06(p>0.05)$, respectively. No systematic under- or overestimation of the QFR was observed when compared with FFR values. Pearson's correlation and agreement between residual QFR and FFR were $0.680, p<0.01$ and $-0.03 \pm$ 0.09 , respectively in Fig. 2A-B. Pearson's correlation was $0.74, p<0.01$ in the EES group (Fig. 2C) and $0.45, p=0.04$ in the BRS group (Fig. 2E) respectively. The agreement was $-0.03 \pm 0.08$ in the EES group (Fig. 2D) and -0.02 \pm 0.12 in the BRS group (Fig. $2 \mathrm{~F}$ ) respectively. The area under the ROC curve for residual QFR to detect a residual FFR of $\leq 0.86$ was 0.869 in all the patients (Fig. 3A). The area under the ROC curve for residual QFR was 0.940 in the EES group (Fig. 3B) and 0.792 in the BRS group (Fig. 3C).

\section{Discussion}

Our study revealed equal diagnostic accuracy of residual QFR in coronary vessels immediately after BRS implantation compared with residual FFR. The residual QFR showed a moderate correlation and agreement with residual FFR for the detection of cutoff 0.86 in post-BRS patients. In addition, the physiologic QFR indices were evaluated using FFR as a reference standard. Importantly, residual QFR showed moderate correlation and certifying per- 
Table 1. Baseline patient characteristics.

\begin{tabular}{lc}
\hline & Total $(73)$ \\
\hline Age (years) & $65.24 \pm 8.98$ \\
Male & $53(70.7 \%)$ \\
BMI $^{1}\left(\mathrm{~kg} / \mathrm{m}^{2}\right)$ & $24.64 \pm 3.16$ \\
Creatinine & $79.76 \pm 18.45$ \\
eGFR & \\
Lp (a) & $87.99 \pm 21.98$ \\
LDL-C & $11.25(5.38,32.50)$ \\
NT Pro BNP & $1.99 \pm 0.78$ \\
HbAlc $(\%)$ & $55.00(26.00,129.00)$ \\
LVEF $^{3}(\%)$ & $6.22 \pm 1.17$ \\
Diabetes & $68.49 \pm 7.92$ \\
Hypertension & $17(22.7 \%)$ \\
Smoking & $40(53.3 \%)$ \\
Prior MI & $22(29.3 \%)$ \\
COPD $^{5}$ & 0 \\
Prior CABG & 0 \\
Prior PCI & \\
EES $^{8}$ & 0 \\
BRS $^{9}$ & $55(73.3 \%)$ \\
\hline${ }^{1}$ Body & $51(70.7 \%)$ \\
\hline
\end{tabular}

\footnotetext{
${ }^{1}$ Body Mass Index; ${ }^{2}$ Endogenous Glomerular Filtration Rate; ${ }^{3}$ Left Ventricular Ejection Fraction; ${ }^{4}$ Myocardial Infarction; ${ }^{5}$ Chronic Obstructive Pulmonary Disease; ${ }^{6}$ Coronary Artery Bypass Surgery; ${ }^{7}$ Percutaneous Coronary Intervention; ${ }^{8}$ Everolimus Eluting Stent; ${ }^{9}$ Bioresorbable Scaffold.
}

formance for invasive FFR, regardless of the types of implanted stents. Residual QFR in the BRS group showed a moderate agreement and correlation compared with the EES group.

There have been numerous efforts to detect the clinic significance of coronary stenoses to determine the need for PCI. Although many non-invasive tests to assess myocardial ischemia are available, previous studies reported a low diagnostic yield [8]. Post-PCI physiologic assessment in complex lesions showed many patients had FFR $<0.8$, especially using OCT/IVUS. However, many patients may still have symptoms after successful PCI, despite an FFR $>0.8$. FFR has been considered the standard invasive method to define the functional significance of coronary stenoses [9]. However, the low utilization rate of FFR may be related to the cost of additional instruments, drugs and equipment, prolongation of operation time, the discomfort of adenosine treatment and the limited confidence in results [10]. In order to overcome these limitations, new non-invasive techniques to evaluate the functional significance of coronary artery stenoses have been developed.

QFR is a method derived from angiography, which calculates the number of 3-dimensional quantitifying coronary angiography (3D QCA) and TIMI (thrombolysis in myocardial infarction) frames without the expense of wire instrumentation or the need for coronary artery hyperemia. A good correlation and agreement were observed between QFR and FFR in the FAVOR Pliot Study [5]. The FAVOR II China Study assessed the diagnostic accuracy of QFR prior to FFR measurement [7]. Compared with diameter stenoses (DS) $\geq 50 \%$ assessed by QCA, the diagnostic accuracy of QFR $\leq 0.80$ is much higher $(92.7 \%$ vs. $59.6 \%$, $p<0.001)$ in the FAVOR II China Study. The FAVOR II Europe-Japan Study assessed the diagnostic accuracy of QFR in 317 vessels from 272 patients with intermediate coronary stenoses [6]. Using FFR as the reference standard, diagnostic accuracy of QFR was $87 \%$. A good correlation ( $\mathrm{r}$ $=0.70, p<0.001$ ) and agreement (mean difference $0.01 \pm$ 0.08 ) were observed between QFR and FFR in the WIFI II Study [12]. Furthermore, QFR significantly correlated with FFR $(\mathrm{r}=0.82, p<0.001)$ with good agreement between QFR and FFR (mean difference $0.011,95 \%$ confidence interval (CI) 0.008-0.015) [13]. Previous studies support the diagnostic value of QFR in assessing the functionality of borderline coronary stenoses and offer a promising alternative for FFR in coronary physiology $[14,15]$.

Although QFR is not currently the gold standard for evaluating myocardial ischemia, QFR is now the most studied angiography-derived FFR technology. Thus, QFR may provide more accurate information on the hemodynamic significance of a coronary stenosis prior to PCI. QFR has not been well validated with FFR in evaluating post-PCI interventions. No studies has been reported on whether QFR is comparable to FFR in patients with residual coronary artery stenoses post-PCI. In this study, we found that QFR showed a good correlation and agreement with FFR in post-PCI both in the EES and BRS groups. This study verifies the improved diagnostic accuracy of QFR with FFR following PCI. The correlation and agreement between residual QFR and FFR were 0.63, $p<0.01$ and $-0.03 \pm 0.09$, respectively. The data was comparable to the results of previous trials [13].

We found moderate correlation and agreement in BRS group. Our study is unique in that it included BRS, the latest generation of coronary stents. QFR and FFR had moderate correlation $(\mathrm{r}=0.45, p=0.04)$ and agreement $(-0.02$ \pm 0.12 ) post-BRS, which has not been reported in previous studies. Although it is very different from metal stents, the performance of BRS in residual QFR is still acceptable. This may broaden the scope of application for QFR, especially for post-PCI evaluation of different stent types.

According to the European Society of Cardiology (ESC) guidelines, FFR is recommended to identify hemodynamically significant coronary lesions [16]. FFR has many limitations in clinical practice. The use of QFR in the catheter laboratory is very feasible [17]. When computed by specialized technicians, the average QFR calculation time is only $4.36 \pm 2.55 \mathrm{~min}$ [18]. In this study, QFR showed a high diagnostic accuracy for the identification of 
FFR $\geq 0.86$ in patients after coronary stent implantation. It can accurately assess coronary artery residual stenoses postBRS implantation using non-invasive technology. The diagnostic accuracy of residual QFR in coronary vessels immediately post-BRS is comparable to FFR.

Visual assessment alone is known to be inaccurate in determining the hemodynamic significance of residual coronary stenoses post-BRS implantation. Additional functional testing, such as FFR increases procedure time and may lead to increased X-ray exposure. Our study showed that residual QFR was similar in accuracy to residual FFR in estimating residual coronary stenoses post-BRS implantation, which decreased operating time and radiation exposure.

\section{Limitations}

This study was limited by its retrospective analysis. Determination of residual coronary stenoses would be more accurate when performed on prospectively selected angiographic projections with high image quality. The selection of angiographic images may result in a superior diagnostic accuracy of residual QFR and a further increase in the proportion of patients that could be correctly deferred from invasive FFR referral post-PCI. Our patient cohort is a realworld representation of the patients that are referred for invasive FFR. Finally, selection bias cannot be excluded due to the retrospective design of this study.

\section{Conclusions}

Residual QFR shows a moderate correlation and good agreement with FFR. Residual QFR assessment after BRS implantation is feasible, and has a moderate correlation and agreement with residual FFR. QFR may be a promising tool similar to FFR to evaluate post-BRS effect.

\section{Abbreviations}

QFR, quantitative flow ratio; FFR, fractional flow reserve; EES, everolimus eluting stent; BRS, bioresorbable scaffold; PCI, percutaneous coronary intervention; ACS, acute coronary syndrome; CABG, coronary artery bypass grafting; ROC, receiver operator characteristic; COPD, obstructive pulmonary diseases; 3D QCA, 3-dimensional quantifying coronary angiography; DS, diameter stenosis; ESC, European Society of Cardiology.

\section{Author contributions}

Conceptualization-ZL and $\mathrm{CJ}$; methodology- JZ and $\mathrm{JH}$; software- $\mathrm{JH}$; validation- $\mathrm{GF}$ and $\mathrm{CJ}$; formal analysis - ZL; investigation - ZL and JZ; resources-ZL and GF; data curation-ZL; writing - original draft preparation-ZL, GF and $\mathrm{CJ}$; writing - review and editing - ZL and $\mathrm{CJ}$; visualization- $\mathrm{CJ}$; supervision $-\mathrm{CJ}$; project administration - $\mathrm{CJ}$; funding acquisition - $\mathrm{ZL}$ and CJ. All authors have read and agreed to the published version of the manuscript.

\section{Ethics approval and consent to participate}

All subjects gave their informed consent for inclusion before they participated in the study. The study was conducted in accordance with the Declaration of Helsinki, and the protocol was approved by the Ethics Committee of Sir Run Run Shaw Hospital Affiliated to Zhejiang University School of Medicine (approval number: 20200917).

\section{Acknowledgment}

Not applicable.

\section{Funding}

This research received no external funding.

\section{Conflict of interest}

The authors declare no conflict of interest.

\section{References}

[1] Madhavan MV, Kirtane AJ, Redfors B, Généreux P, BenYehuda O, Palmerini T, et al. Stent-Related Adverse Events $>1$ Year after Percutaneous Coronary Intervention. Journal of the American College of Cardiology. 2020; 75: 590-604.

[2] Van Belle E, Cosenza A, Baptista SB, Vincent F, Henderson J, Santos L, et al. Usefulness of Routine Fractional Flow Reserve for Clinical Management of Coronary Artery Disease in Patients with Diabetes. JAMA Cardiology. 2020; 5: 272-281.

[3] De Bruyne B, Pijls NHJ, Kalesan B, Barbato E, Tonino PAL, Piroth Z, et al. Fractional Flow Reserve-Guided PCI versus Medical Therapy in Stable Coronary Disease. New England Journal of Medicine. 2012; 367: 991-1001.

[4] . Baumann S, Bojara W, Post H, Rudolph T, Schaufele T, Ong $\mathrm{P}$, et al. Coronary physiology in the catheter laboratory. Herz. 2020; 46: 15-23. (In German)

[5] Tu S, Westra J, Yang J, von Birgelen C, Ferrara A, Pellicano M, et al. Diagnostic Accuracy of Fast Computational Approaches to Derive Fractional Flow Reserve from Diagnostic Coronary Angiography. JACC: Cardiovascular Interventions. 2016; 9: 2024 2035.

[6] Westra J, Andersen BK, Campo G, Matsuo H, Koltowski L, Eftekhari A, et al. Diagnostic Performance of In-Procedure Angiography-Derived Quantitative Flow Reserve Compared to Pressure-Derived Fractional Flow Reserve: The FAVOR II Europe-Japan Study. Journal of the American Heart Association. 2018; 7: e009603.

[7] Xu B, Tu S, Qiao S, Qu X, Chen Y, Yang J, et al. Diagnostic Accuracy of Angiography-Based Quantitative Flow Ratio Measurements for Online Assessment of Coronary Stenosis. Journal of the American College of Cardiology. 2017; 70: 3077-3087.

[8] Xu B, Tu S, Song L, Jin Z, Yu B, Fu G, et al. Angiographic quantitative flow ratio-guided coronary intervention (FAVOR III China): a multicentre, randomised, sham-controlled trial. The Lancet. 2021; 398: 2149-2159.

[9] Rubimbura V, Guillon B, Fournier S, Amabile N, Chi Pan C, Combaret $\mathrm{N}$, et al. Quantitative flow ratio virtual stenting and post stenting correlations to post stenting fractional flow reserve measurements from the DOCTORS (does Optical Coherence Tomography Optimize Results of Stenting) study population. Catheterization and Cardiovascular Interventions. 2020; 96: $1145-1153$.

[10] Xu K, Fu G, Xu B, Zhou Y, Su X, Liu H, et al. Safety and efficacy of the novel sirolimus-eluting bioresorbable scaffold for the treatment of de novo coronary artery disease: One-year results from a prospective patient-level pooled analysis of NeoVas 
trials. Catheterization and Cardiovascular Interventions. 2019; 93: 832-838.

[11] Ties D, van Dijk R, Pundziute G, Lipsic E, Vonck TE, van den Heuvel AFM, et al. Computational quantitative flow ratio to assess functional severity of coronary artery stenosis. International Journal of Cardiology. 2018; 271: 36-41.

[12] Johnson NP, Koo B. Coronary Psychology: do you Believe? JACC: Cardiovascular Interventions. 2018; 11: 1492-1494.

[13] Stähli BE, Erbay A, Steiner J, Klotsche J, Mochmann HC, Skurk $\mathrm{C}$, et al. Comparison of resting distal to aortic coronary pressure with angiography-based quantitative flow ratio. International Journal of Cardiology. 2019; 279: 12-17.

[14] Kleczyński P, Dziewierz A, Rzeszutko Ł, Dudek D, Legutko J. Contrast medium $\mathrm{Pd} / \mathrm{Pa}$ ratio in comparison to fractional flow reserve, quantitative flow ratio and instantaneous wave-free ratio for evaluation of intermediate coronary lesions. Postępy w Kardiologii Interwencyjnej. 2020; 16: 384-390.
[15] Kleczyński P, Dziewierz A, Rzeszutko L, Dudek D, Legutko J. Is quantitative flow ratio enough to accurately assess intermediate coronary stenosis? A comparison study with fractional flow reserve. Cardiology Journal. 2019; 26: 793-795.

[16] Neumann FJ, Sousa-Uva M, Ahlsson A, Alfonso F, Banning AP, Benedetto U, et al. $2018 \mathrm{ESC} / \mathrm{EACTS}$ Guidelines on myocardial revascularization. European Heart Journal. 2019; 40: 87-165.

[17] Buono A, Mühlenhaus A, Schäfer T, Trieb AK, Schmeißer J, Koppe F, et al. QFR Predicts the Incidence of Long-Term Adverse Events in Patients with Suspected CAD: Feasibility and Reproducibility of the Method. Journal of Clinical Medicine. 2020; 9: 220.

[18] Xu B, Tu S, Qiao S, Qu X, Chen Y, Yang J, et al. Diagnostic Accuracy of Angiography-Based Quantitative Flow Ratio Measurements for Online Assessment of Coronary Stenosis. Journal of the American College of Cardiology. 2017; 70: 3077-3087. 